\title{
SURFACE PENCIL WITH A COMMON LINE OF CURVATURE IN MINKOWSKI 3-SPACE
}

\author{
Evren ERGÜN, Ergin BAYRAM, Emin KASAP \\ Ondokuz Mayıs University, Faculty of Arts and Sciences, \\ Department of Mathematics, Samsun TURKEY \\ eergun@omu.edu.tr, erginbayram@yahoo.com, kasape@omu.edu.tr
}

\begin{abstract}
In this paper, we analyze the problem of constructing a surface pencil from a given spacelike (timelike) line of curvature. By using the Frenet frame of the given curve in Minkowski 3-space, we express the surface pencil as a linear combination of this frame and derive the necessary and sufficient conditions for the coefficients to satisfy the line of curvature requirement. To illustrate the method some examples showing members of the surface pencil with their line of curvature are given.
\end{abstract}

Keywords: Minkowski space, Line of curvature, Surface pencil.

2000 Mathematics Subject Classification: 53B30, 51B20, 53C50.

Short Title: Surfaces with common line of curvature in $\mathbb{R}_{1}^{3}$ 


\section{Introduction}

On a Minkowski surface, tangent vectors are classified into timelike, spacelike or null and so a curve on the surface is said to be timelike, spacelike or null if its tangent vectors are always timelike, spacelike or null, respectively. In fact, a timelike curve corresponds to the path of an observer moving slower than the speed of light, spacelike curve corresponds to moving faster than the speed of light and null curve corresponds to moving at the speed of light. Surface with a common characteristic curve have been the subject of many recent studies. Wang et.al.[1] studied the problem of constructing a surface pencil from a given spatial geodesic. They parametrized the surface by using the Frenet frame of the given curve and gave the necessary and sufficient condition to satify the geodesic requirement. Kasap and Akyıldız [2] considered the surfaces with common geodesic in Minkowski 3-space. They studied spacelike surface with common spacelike geodesic and timelike surface with common spacelike or timelike geodesic. Li et.al.[3] derived the necessary and sufficient condition for a given curve to be a line of curvature on a surface. Şaffak and Kasap [4] analyzed the problem of finding a surface family through a null geodesic with Cartan frame. Being inspired by the above studies, we extend the method of Lie et. al. [3] to derive the necessary and sufficient condition for the given curve to be a line of curvature for the parametric surface. By utilizing the Frenet

frame, we derive necessary and sufficient condition for the correct parametric representation of the surface $P(s, t)$ when the parameter $s$ is the arc-length of the curve $r(s)$ and find the necessary constraints on the coefficients of vectors of the frame ( which are called marching-scale functions) so that both the line of curvature and parametric requirement are met. Thus, we defined the spacelike and timelike surface pencil with common line of curvature. Also, we give two general forms of the marching-scale functions and obtain necessary and suffi- 
cient conditions on them for which the given curve is a line of curvature on a surface. Finally, we give some nice examples, showing members of the surface pencil with their common line of curvatures to illustrate the method.

\section{Preliminaries}

Let us consider Minkowski 3-space $\mathbb{R}_{1}^{3}=\left[\mathbb{R}^{3},(+,+,-)\right]$ and let the Lorentzian inner product of $X=\left(x_{1}, x_{2}, x_{3}\right)$ and $Y=\left(y_{1}, y_{2}, y_{3}\right) \in \mathbb{R}_{1}^{3}$ be

$$
\langle X, Y\rangle=x_{1} y_{1}+x_{2} y_{2}-x_{3} y_{3} .
$$

A vector $X \in \mathbb{R}_{1}^{3}$ is called a spacelike vector when $\langle X, X\rangle>0$ or $X=0$. It is called timelike and null (lightlike) vector in case of $\langle X, X\rangle\langle 0,\langle X, X\rangle=0$ for $X \neq 0$.respectively, [5]. The vector product of vectors $X=\left(x_{1}, x_{2}, x_{3}\right)$ and $Y=\left(y_{1}, y_{2}, y_{3}\right) \in \mathbb{R}_{1}^{3}$ is defined by $[6]$

$$
X \times Y=\left(x_{2} y_{3}-x_{3} y_{2}, x_{3} y_{1}-x_{1} y_{3}, x_{2} y_{1}-x_{1} y_{2}\right) .
$$

Let $r=r(s)$ be a unit speed curve in $\mathbb{R}_{1}^{3}$. By $\kappa(s)$ and $\tau(s)$ we denote the natural curvature and torsion of $r(s)$,respectively. Consider the Frenet frame $\{T, N, B\}$ associated with the curve $r=r(s)$ such that $T=T(s), N=$ $N(s)$ and $B=B(s)$ are the unit tangent, the principal normal and the binormal vector fields, respectively. If $r=r(s)$ is a spacelike curve, then the structural equations (or Frenet formulas) of this frame are given as

$$
\dot{T}(s)=\kappa(s) N(s), \dot{N}(s)=\varepsilon \kappa(s) T(s)+\tau(s) B(s), \dot{B}(s)=\tau(s) N(s)
$$


where

$$
\varepsilon=\left\{\begin{array}{l}
+1, B \text { is spacelike } \\
-1, B \text { is timelike }
\end{array}\right.
$$

If $r=r(s)$ is a timelike curve, then above equations are given as [7]

$$
\dot{T}(s)=\kappa(s) N(s), \dot{N}(s)=\kappa(s) T(s)-\tau(s) B(s), \dot{B}(s)=\tau(s) N(s) .
$$

The norm of a vector $X$ is defined by [5]

$$
\|X\|_{I L}=\sqrt{|\langle X, X\rangle|}
$$

Theorem 1 : Let $X$ and $Y$ be non-zero orthogonal Lorentz vectors in $\mathbb{R}_{1}^{3}$. If $X$ is timelike, then $Y$ is spacelike [8].

Theorem 2 : Let $X$ and $Y$ be positive (negative) timelike vectors in $\mathbb{R}_{1}^{3}$. Then

$$
\langle X, Y\rangle \leq\|X\|\|Y\|
$$

with equality if and only if $X$ and $Y$ are linearly dependent [8].

Let $X$ and $Y$ be positive (negative ) timelike vectors in $\mathbb{R}_{1}^{3}$. Then there is a unique non-negative real number $\varphi(X, Y)$ such that

$$
\langle X, Y\rangle=\|X\|\|Y\| \cosh \varphi(X, Y)
$$

the Lorentzian timelike angle between $X$ and $Y$ is defined to be $\varphi(X, Y)[8]$.

Let $X$ and $Y$ be spacelike vectors in $\mathbb{R}_{1}^{3}$ that span a spacelike vector subspace. Then we have

$$
|\langle X, Y\rangle| \leq\|X\|\|Y\|
$$

with equality if and only if $X$ and $Y$ are linearly dependent. Hence, there is a 
unique real number $\varphi(X, Y)$ between 0 and $\pi$ such that

$$
\langle X, Y\rangle=\|X\|\|Y\| \cos \varphi(X, Y)
$$

the Lorentzian spacelike angle between $X$ and $Y$ is defined to be $\varphi(X, Y)$ [8].

Let $X$ and $Y$ be spacelike vectors in $\mathbb{R}_{1}^{3}$ that span a timelike vector subspace. Then we have

$$
|\langle X, Y\rangle|>\|X\|\|Y\|
$$

Hence, there is a unique positive real number $\varphi(X, Y)$ between 0 and $\pi$ such that

$$
|\langle X, Y\rangle|=\|X\|\|Y\| \cosh \varphi(X, Y)
$$

the Lorentzian timelike angle between $X$ and $Y$ is defined to be $\varphi(X, Y)[8]$.

Let $X$ be a spacelike vector and $Y$ be a positive timelike vector in $\mathbb{R}_{1}^{3}$. Then there is a unique nonnegative real number $\varphi(X, Y)$ such that

$$
|\langle X, Y\rangle|=\|X\|\|Y\| \sinh \varphi(X, Y)
$$

the Lorentzian timelike angle between $X$ and $Y$ is defined to be $\varphi(X, Y)$ [8].

Let $M$ be a semi-Riemannian submanifold of $\bar{M}$ and $\bar{D}$ be the Levi-Civita connection of $\bar{M}$, the function $I I: \chi(M) \times \chi(M) \longrightarrow \chi(M)^{\perp}$ such that

$$
I I(X, Y)=\operatorname{nor} \bar{D}_{X} Y
$$

is $\Im(M)$-bilinear and symmetric. $I I$ is called the shape tensor (or second fundamental form tensor) of $M \subset \bar{M}[5]$.

Let $N$ be a unit normal vector field on a semi-Riemannian hypersurface 
$M \subset \bar{M}$. The $(1,1)$ tensor field $S$ on $M$ such that

$$
\langle S(X), Y\rangle=\langle I I(X, Y), N\rangle
$$

for all $X, Y \in \chi(M)$ is called the shape operator of $M \subset \bar{M}$ derived from $N$ [5].

As usual, $S$ determines a linear operator $S: T_{P}(M) \longrightarrow T_{P}(M)$ at each point $P \in M$. If $S$ is shape operator derived from $N$, then $S(X)=-\bar{D}_{X} N$ and at each point the linear operator $S$ on $T_{P}(M)$ is self adjoint. A regular curve $\mathrm{r}$ on $M$ is said to be a line of curvature of $M$ if for all $p \in r$ the tangent line of $r$ is a principal direction at $p$. According to this definition, the differential equation of the line of curvature on $M$ is $S(T)=\omega T, \omega \neq 0$, where $S$ is the shape operator of $M$.

A surface in $\mathbb{R}_{1}^{3}$ is called a timelike surface if the induced metric on the surface is a Lorentzian metric and is called a spacelike surface if induced metric on the surface is positive definite Riemannian metric, i.e. the normal vector on the spacelike (timelike) surface is a timelike (spacelike) vector [9].

A parametric curve $r(s)$ is a curve on a surface $P=P(s, t)$ in $\mathbb{R}_{1}^{3}$ that has a constant $s$ or $t$ parameter value, that is, there exists a parameter $s_{0}$ or $t_{0}$ such that

$$
r(s)=P\left(s, t_{0}\right) \text { or } r(t)=P\left(s_{0}, t\right) .
$$




\section{Spacelike Surface Pencil with a Common Line of Curvature}

Let $P=P(s, t)$ be a parametric spacelike surface and $r=r(s)$ be a spacelike curve with spacelike binormal. The surface is defined by the given curve as

$$
P(s, t)=r(s)+(u(s, t), v(s, t), w(s, t))\left(\begin{array}{c}
T(s) \\
N(s) \\
B(s)
\end{array}\right),
$$

$L_{1} \leq s \leq L_{2}, T_{1} \leq t \leq T_{2}$, where $u(s, t), v(s, t)$ and $w(s, t)$ are called the marching-scale functions and $\{T(s), N(s), B(s)\}$ is the Frenet frame associated with the curve $r(s)$.

Since the curve $r(s)$ is a parametric curve on the surface $P(s, t)$, there exists a parameter $t_{0} \in\left[T_{1}, T_{2}\right]$ such that $P\left(s, t_{0}\right)=r(s), L_{1} \leq s \leq L_{2}$, that is,

$$
u\left(s, t_{0}\right)=v\left(s, t_{0}\right)=w\left(s, t_{0}\right) \equiv 0, L_{1} \leq s \leq L_{2} .
$$

Let $n_{1}\left(n_{1}=\cosh \theta N+\sinh \theta B\right)$ be a vector orthogonal to the curve $r(s)$, where $\theta=\theta(s)$ is the Lorentzian timelike angle between $N$ and $n_{1}$. The curve $r(s)$ is a line of curvature on the surface $P(s, t)$ if and only if $n_{1}$ is parallel to the normal vector $n(s, t)$ of the surface $P(s, t)$ and $S(T)=\omega T, \omega \neq 0$, where $S$ is the shape operator of the surface.

Firstly, we derive the condition for $n_{1}$ to be parallel to the normal vector $n(s, t)$ of the surface $P(s, t)$ :

The normal vector can be expressed as

$$
\begin{aligned}
& n(s, t)=\frac{\partial P(s, t)}{\partial s} \times \frac{\partial P(s, t)}{\partial t} \\
& =\left(-\left(\frac{\partial w(s, t)}{\partial s}+v(s, t) \tau(s)\right) \frac{\partial v(s, t)}{\partial t}+\right. \\
& \left.\left(\frac{\partial v(s, t)}{\partial s}+u(s, t) \kappa(s)+w(s, t) \tau(s)\right) \frac{\partial w(s, t)}{\partial t}\right) T(s)+
\end{aligned}
$$




$$
\begin{aligned}
& \left(\left(1+\frac{\partial u(s, t)}{\partial s}+v(s, t) \kappa(s)\right) \frac{\partial w(s, t)}{\partial t}-\right. \\
& \left.\left(\frac{\partial w(s, t)}{\partial s}+v(s, t) \tau(s)\right) \frac{\partial u(s, t)}{\partial t}\right) N(s)+ \\
& \left(\left(1+\frac{\partial u(s, t)}{\partial s}+v(s, t) \kappa(s)\right) \frac{\partial v(s, t)}{\partial t}-\right. \\
& \left.\left(\frac{\partial v(s, t)}{\partial s}+u(s, t) \kappa(s)+w(s, t) \tau(s)\right) \frac{\partial u(s, t)}{\partial t}\right) B(s)
\end{aligned}
$$

Thus, we get

$$
n\left(s, t_{0}\right)=\phi_{1}\left(s, t_{0}\right) T(s)+\phi_{2}\left(s, t_{0}\right) N(s)+\phi_{3}\left(s, t_{0}\right) B(s),
$$

where

$$
\begin{aligned}
& \phi_{1}\left(s, t_{0}\right)=\frac{\partial v\left(s, t_{0}\right)}{\partial s} \frac{\partial w\left(s, t_{0}\right)}{\partial t}-\frac{\partial w\left(s, t_{0}\right)}{\partial s} \frac{\partial v\left(s, t_{0}\right)}{\partial t} \\
& \phi_{2}\left(s, t_{0}\right)=\left(1+\frac{\partial u\left(s, t_{0}\right)}{\partial s}\right) \frac{\partial w\left(s, t_{0}\right)}{\partial t}-\frac{\partial w\left(s, t_{0}\right)}{\partial s} \frac{\partial u\left(s, t_{0}\right)}{\partial t} \\
& \phi_{3}\left(s, t_{0}\right)=\left(1+\frac{\partial u\left(s, t_{0}\right)}{\partial s}\right) \frac{\partial v\left(s, t_{0}\right)}{\partial t}-\frac{\partial v\left(s, t_{0}\right)}{\partial s} \frac{\partial u\left(s, t_{0}\right)}{\partial t} .
\end{aligned}
$$

This follows that $n_{1}(s) / / n\left(s, t_{0}\right), L_{1} \leq s \leq L_{2}$, if and only if there exits a function $\lambda(s) \neq 0$ such that

$$
\phi_{1}\left(s, t_{0}\right)=0, \phi_{2}\left(s, t_{0}\right)=\lambda(s) \cosh \theta, \phi_{3}\left(s, t_{0}\right)=\lambda(s) \sinh \theta .
$$

Secondly, since $S(T)=\omega T, \omega \neq 0$, we obtain

$$
\theta(s)=-\int_{s_{0}}^{s} \tau d s+\theta_{0}
$$

where $s_{0}$ is the starting value of the arc-length and $\theta=\theta(s)$. In this paper, we assume that $s_{0}=0$.

Combining (3.2), (3.3) and (3.4), we have the following theorem.

Theorem 3 A spacelike curve $r(s)$ with spacelike binormal is a line of curvature on the surface $P(s, t)$ if and only if the followings are satisfied:

$$
\theta(s)=-\int_{s_{0}}^{s} \tau d s+\theta(0)
$$




$$
\begin{aligned}
& u\left(s, t_{0}\right)=v\left(s, t_{0}\right)=w\left(s, t_{0}\right) \equiv 0, \\
& \phi_{1}\left(s, t_{0}\right) \equiv 0, \phi_{2}\left(s, t_{0}\right)=\lambda(s) \cosh \theta, \phi_{3}\left(s, t_{0}\right)=\lambda(s) \sinh \theta .
\end{aligned}
$$

We call the set of surfaces defined by (3.1) - (3.4) spacelike surface pencil with a common line of curvature. Any surface $P(s, t)$ defined by $(3.1)$ and satisfying $(3.2)-(3.4)$ is a member of this family.

Now, we analyse two different types of the marching-scale functions $u(s, t), v(s, t)$ and $w(s, t)$ in. the Eq. (3.1).

(i) If we choose

$$
\begin{aligned}
& u(s, t)=\sum_{k=1}^{p} a_{1 k} l(s)^{k} U(t)^{k}, v(s, t)=\sum_{k=1}^{p} a_{2 k} m(s)^{k} V(t)^{k} \text { and } w(s, t)= \\
& \sum_{k=1}^{p} a_{3 k} n(s)^{k} W(t)^{k}
\end{aligned}
$$

then, we can simply express the sufficient condition for which the curve $r(s)$ is a line of curvature of the surface $P(s, t)$ as

$$
\begin{aligned}
U\left(t_{0}\right) & =V\left(t_{0}\right)=W\left(t_{0}\right)=0 \\
\theta(s) & =-\int_{s_{0}}^{s} \tau d s+\theta_{0} \\
a_{21} m(s) V^{\prime}\left(t_{0}\right) & =\lambda(s) \sinh \theta, a_{31} n(s) W^{\prime}\left(t_{0}\right)=\lambda(s) \cosh \theta
\end{aligned}
$$

$\lambda(s) \neq 0$, where $l(s), m(s), n(s), U(t), V(t)$ and $W(t)$ are $C^{1}$ functions, $a_{i j} \in \mathbb{R}(k=1,2,3 ; j=1,2,3, \ldots, p)$.

(ii) If we choose

$$
\begin{aligned}
& u(s, t)=f\left(\sum_{k=1}^{p} a_{1 k} l(s)^{k} U(t)^{k}\right), \quad v(s, t)=g\left(\sum_{k=1}^{p} a_{2 k} m(s)^{k} V(t)^{k}\right) \text { and } \\
& w(s, t)=h\left(\sum_{k=1}^{p} a_{3 k} n(s)^{k} W(t)^{k}\right)
\end{aligned}
$$

then, we can express the sufficient condition for which the curve $r(s)$ is a line of curvature on the surface $P(s, t)$ as 


$$
\begin{aligned}
U\left(t_{0}\right) & =V\left(t_{0}\right)=W\left(t_{0}\right)=0 \text { and } f(0)=g(0)=h(0) \\
\theta(s) & =-\int_{s_{0}}^{s} \tau d s+\theta_{0} \\
g^{\prime}(0) a_{21} m(s) V^{\prime}\left(t_{0}\right) & =\lambda(s) \sinh \theta, h^{\prime}(0) a_{31} n(s) W^{\prime}\left(t_{0}\right)=\lambda(s) \cosh \theta
\end{aligned}
$$

$\lambda(s) \neq 0$, where $l(s), m(s), n(s), U(t), V(t)$ and $W(t)$ are $C^{1}$ functions, $a_{i j} \in \mathbb{R}(k=1,2,3 ; j=1,2,3, \ldots, p)$.

Example 4 Let $r(s)=\left(a \sinh \left(\frac{s}{c}\right), \frac{b s}{c}, a \cosh \left(\frac{s}{c}\right)\right)$ be a spacelike curve,

$a, b, c \in \mathbb{R}, a^{2}+b^{2}=c^{2}$ and $-2 \leq s \leq 2$. It is easy to show that

$T(s)=\left(\frac{a}{c} \cosh \left(\frac{s}{c}\right), \frac{b}{c}, \frac{a}{c} \sinh \left(\frac{s}{c}\right)\right)$,

$N(s)=\left(\sinh \left(\frac{s}{c}\right), 0, \cosh \left(\frac{s}{c}\right)\right)$,

$B(s)=\left(\frac{b}{c} \cosh \left(\frac{s}{c}\right),-\frac{a}{c}, \frac{b}{c} \sinh \left(\frac{s}{c}\right)\right)$.

By taking $\theta(0)=0$ we have $\theta(s)=-\frac{b s}{c^{2}}$. If we choose $\lambda(s) \equiv 1, t_{0}=$

$0, a_{21}=a_{31}=1$ and

$$
\begin{aligned}
& u(s, t)=\sum_{k=1}^{3} a_{1 k} l(s) U(t) \equiv 0, \\
& v(s, t)=\sinh \left(-\frac{b s}{c^{2}}\right) t+\sum_{k=2}^{3} a_{2 k} \sinh ^{k}\left(-\frac{b s}{c^{2}}\right) t^{k}, \\
& w(s, t)=\cosh \left(-\frac{b s}{c^{2}}\right) t+\sum_{k=2}^{3} a_{3 k} \cosh ^{k}\left(-\frac{b s}{c^{2}}\right) t^{k}
\end{aligned}
$$

then the Eq. (3.5) is satisfied.

Letting $a=b=1$, we immediately obtain a member of the surface pencil

(Fig. 3.1) as

$$
\begin{aligned}
& P_{1}(s, t)=\left(\sinh \left(\frac{s}{\sqrt{2}}\right)+\sinh \left(-\frac{s}{2}\right) t \sinh \left(\frac{s}{\sqrt{2}}\right)+\sinh \left(\frac{s}{\sqrt{2}}\right) \sum_{k=2}^{3} a_{2 k} \sinh ^{k}\left(-\frac{s}{2}\right) t^{k}+\right. \\
& \frac{\sqrt{2}}{2} \cosh \left(-\frac{s}{2}\right) t \cosh \left(\frac{s}{\sqrt{2}}\right)+\frac{\sqrt{2}}{2} \cosh \left(\frac{s}{\sqrt{2}}\right) \sum_{k=2}^{3} a_{3 k} \cosh ^{k}\left(-\frac{s}{2}\right) t^{k}, \\
& \quad \frac{s}{\sqrt{2}}-\frac{\sqrt{2}}{2} \cosh \left(-\frac{s}{2}\right) t-\frac{\sqrt{2}}{2} \sum_{k=2}^{3} a_{3 k} \cosh ^{k}\left(-\frac{s}{2}\right) t^{k}, \cosh \left(\frac{s}{\sqrt{2}}\right)+\sinh \left(-\frac{s}{2}\right) t \cosh \left(\frac{s}{\sqrt{2}}\right)+ \\
& \cosh \left(\frac{s}{\sqrt{2}}\right) \sum_{k=2}^{3} a_{2 k} \sinh ^{k}\left(-\frac{s}{2}\right) t^{k}
\end{aligned}
$$




$$
\left.+\frac{\sqrt{2}}{2} \cosh \left(-\frac{s}{2}\right) t \sinh \left(\frac{s}{\sqrt{2}}\right)+\frac{\sqrt{2}}{2} \sinh \left(\frac{s}{\sqrt{2}}\right) \sum_{k=2}^{3} a_{3 k} \cosh ^{k}\left(-\frac{s}{2}\right) t^{k}\right) .
$$

Fig. 3.1. $P_{1}(s, t)$ as a member of the surface pencil and its line of curvature

Example 5 Let $r(s)=\left(\frac{\sqrt{3}}{2} \sinh (s), \frac{s}{2}, \frac{\sqrt{3}}{2} \cosh (s)\right)$ be an arc-length spacelike curve, $0 \leq s \leq 2 \pi$. It is easy to show that

$$
\begin{aligned}
& T(s)=\left(\frac{\sqrt{3}}{2} \cosh (s), \frac{1}{2}, \frac{\sqrt{3}}{2} \sinh (s)\right), \\
& N(s)=(\sinh (s), 0, \cosh (s)), \\
& B(s)=\left(\frac{1}{2} \cosh (s),-\frac{\sqrt{3}}{2}, \frac{1}{2} \sinh (s)\right), \\
& \tau=\frac{1}{2} .
\end{aligned}
$$

Taking $\theta(0)=0$ we have $\theta(s)=-\frac{1}{2} s$. If we choose $\lambda(s) \equiv 1, t_{0}=0, a_{21}=$ $a_{31}=1$ and

$$
u(s, t)=t, v(s, t)=\sinh \left(-\frac{1}{2} s\right) t, w(s, t)=\cosh \left(-\frac{1}{2} s\right) t
$$

then the Eq. (3.5) is satisfied. So, we have the following surface as a member of the surface pencil with common line of curvature $r(s)$ (Fig. 3.2) as

$$
\begin{aligned}
& P_{2}(s, t)=\left(\frac{\sqrt{3}}{2} \sinh (s)+\frac{\sqrt{3}}{2} t \cosh (s)+t \cosh \left(-\frac{1}{2} s\right) \sinh (s)-t \sinh \left(-\frac{1}{2} s\right) \cosh \left(-\frac{1}{2} s\right),\right. \\
& \frac{s}{2}+\frac{t}{2}+\frac{\sqrt{3}}{2},
\end{aligned}
$$


$\left.\frac{\sqrt{3}}{2} \cosh (s)+\frac{\sqrt{3}}{2} t \sinh (s)+\cosh \left(-\frac{1}{2} s\right) \cosh (s)-\frac{1}{2} \sinh \left(-\frac{1}{2} s\right) \sinh (s)\right)$

where $0 \leq s \leq 2 \pi, \quad-2 \leq t \leq 2$

Fig. 3.2. $P_{2}(s, t)$ as a member of the surface pencil and its line of curvature

\section{Timelike Surface Pencil with a Common Line of Curvature}

Let $P=P(s, t)$ be a parametric timelike surface and $r=r(s)$ be a spacelike curve with timelike binormal. The surface is defined by the given curve as

$$
P(s, t)=r(s)+(u(s, t), v(s, t), w(s, t))\left(\begin{array}{c}
T(s) \\
N(s) \\
B(s)
\end{array}\right)
$$

$L_{1} \leq s \leq L_{2}, T_{1} \leq t \leq T_{2}$, where $\{T(s), N(s), B(s)\}$ is the Frenet frame associated with the curve $r(s)$.

Since the curve $r(s)$ is a parametric curve on the surface $P(s, t)$, there exists 
a parameter $t_{0} \in\left[T_{1}, T_{2}\right]$ such that $P\left(s, t_{0}\right)=r(s) L_{1} \leq s \leq L_{2}$, that is ,

$$
u\left(s, t_{0}\right)=v\left(s, t_{0}\right)=w\left(s, t_{0}\right) \equiv 0, L_{1} \leq s \leq L_{2} .
$$

Let $n_{1}\left(n_{1}=\cosh \theta N+\sinh \theta B\right)$ be a vector orthogonal to the curve $r(s)$, where $\theta=\theta(s)$ is the Lorentzian timelike angle between $N$ and $n_{1}$. The curve $r(s)$ is a line of curvature on the surface $P(s, t)$ if and only if $n_{1}$ is parallel to the normal vector $n(s, t)$ of the surface $P(s, t)$ and $S(T)=\omega T, \omega \neq 0$, where $S$ is the shape operator of the surface.

Firstly, we derive the condition for $n_{1}$ to be parallel to the normal vector $n(s, t)$ of the surface $P(s, t)$ :

The normal vector can be expressed as

$$
\begin{aligned}
& n(s, t)=\frac{\partial P(s, t)}{\partial s} \times \frac{\partial P(s, t)}{\partial t} \\
& =\left(-\left(\tau(s) v(s, t)+\frac{\partial w(s, t)}{\partial s}\right) \frac{\partial v(s, t)}{\partial t}+\right. \\
& \left.\left(-\kappa(s) u(s, t)+\tau(s) w(s, t)+\frac{\partial v(s, t)}{\partial s}\right) \frac{\partial w(s, t)}{\partial t}\right) T(s)+ \\
& \left(\left(\tau(s) v(s, t)+\frac{\partial w(s, t)}{\partial s}\right) \frac{\partial u(s, t)}{\partial t}-\right. \\
& \left.\left(1-\kappa(s) v(s, t)+\frac{\partial u(s, t)}{\partial s}\right) \frac{\partial w(s, t)}{\partial t}\right) N(s)+ \\
& \left(\left(-\kappa(s) u(s, t)+\tau(s) w(s, t)+\frac{\partial v(s, t)}{\partial s}\right) \frac{\partial u(s, t)}{\partial t}-\right. \\
& \left.\left(1-\kappa(s) v(s, t)+\frac{\partial u(s, t)}{\partial s}\right) \frac{\partial v(s, t)}{\partial t}\right) B(s) .
\end{aligned}
$$

Thus, we get

$$
n\left(s, t_{0}\right)=\phi_{1}\left(s, t_{0}\right) T(s)+\phi_{2}\left(s, t_{0}\right) N(s)+\phi_{3}\left(s, t_{0}\right) B(s)
$$

where

$$
\begin{aligned}
& \phi_{1}\left(s, t_{0}\right)=\frac{\partial v\left(s, t_{0}\right)}{\partial s} \frac{\partial w\left(s, t_{0}\right)}{\partial t}-\frac{\partial w\left(s, t_{0}\right)}{\partial s} \frac{\partial v\left(s, t_{0}\right)}{\partial t}, \\
& \phi_{2}\left(s, t_{0}\right)=-\left(1+\frac{\partial u\left(s, t_{0}\right)}{\partial s}\right) \frac{\partial w\left(s, t_{0}\right)}{\partial t}+\frac{\partial w\left(s, t_{0}\right)}{\partial s} \frac{\partial u\left(s, t_{0}\right)}{\partial t}, \\
& \phi_{3}\left(s, t_{0}\right)=-\left(1+\frac{\partial u\left(s, t_{0}\right)}{\partial s}\right) \frac{\partial v\left(s, t_{0}\right)}{\partial t}+\frac{\partial v\left(s, t_{0}\right)}{\partial s} \frac{\partial u\left(s, t_{0}\right)}{\partial t} .
\end{aligned}
$$

This follows that $n_{1}(s) / / n\left(s, t_{0}\right), L_{1} \leq s \leq L_{2}$, if and only if there exits a 
function $\lambda(s) \neq 0$ such that

$$
\phi_{1}\left(s, t_{0}\right)=0, \phi_{2}\left(s, t_{0}\right)=\lambda(s) \cosh \theta, \phi_{3}\left(s, t_{0}\right)=\lambda(s) \sinh \theta .
$$

Secondly, since $S(T)=\omega T, \omega \neq 0$,

$$
\theta(s)=-\int_{s_{0}}^{s} \tau d s+\theta_{0},
$$

where $s_{0}$ is the starting value of arc length and $\theta=\theta(s)$. In this paper, we assume $s_{0}=0$.

Combining (4.2), (4.3) and (4.4), we have the following theorem.

Theorem 6 A spacelike curve $r(s)$ with timelike binormal is a line of curvature on the surface $P(s, t)$ if and only if the followings are satisfied

$$
\begin{aligned}
& \theta(s)=-\int_{s_{0}}^{s} \tau d s+\theta(0), \\
& u\left(s, t_{0}\right)=v\left(s, t_{0}\right)=w\left(s, t_{0}\right) \equiv 0, \\
& \phi_{1}\left(s, t_{0}\right) \equiv 0, \phi_{2}\left(s, t_{0}\right)=\lambda(s) \cosh \theta, \phi_{3}\left(s, t_{0}\right)=\lambda(s) \sinh \theta .
\end{aligned}
$$

Now, we analyse two different types of the marching-scale functions $u(s, t)$, $v(s, t)$ and $w(s, t)$ in the Eq. (4.1).

(i) If we choose

$$
\begin{aligned}
& u(s, t)=\sum_{k=1}^{p} a_{1 k} l(s)^{k} U(t)^{k}, v(s, t)=\sum_{k=1}^{p} a_{2 k} m(s)^{k} V(t)^{k} \text { and } w(s, t)= \\
& \sum_{k=1}^{p} a_{3 k} n(s)^{k} W(t)^{k} \\
& \text { then, we can simply express the sufficient condition for which the curve } r(s)
\end{aligned}
$$
is a line of curvature on the surface $P(s, t)$ as 


$$
\begin{aligned}
U\left(t_{0}\right) & =V\left(t_{0}\right)=W\left(t_{0}\right)=0 \\
\theta(s) & =-\int_{s_{0}}^{s} \tau d s+\theta_{0}, \\
a_{21} m(s) V^{\prime}\left(t_{0}\right) & =-\lambda(s) \sinh \theta, a_{31} n(s) W^{\prime}\left(t_{0}\right)=-\lambda(s) \cosh \theta,
\end{aligned}
$$

$\lambda(s) \neq 0$, where $l(s), m(s), n(s), U(t), V(t)$ and $W(t)$ are $C^{1}$ functions, $a_{i j} \in \mathbb{R}(k=1,2,3 ; j=1,2,3, \ldots, p)$.

(ii) If we choose

$u(s, t)=f\left(\sum_{k=1}^{p} a_{1 k} l(s)^{k} U(t)^{k}\right), v(s, t)=g\left(\sum_{k=1}^{p} a_{2 k} m(s)^{k} V(t)^{k}\right)$ and $w(s, t)=h\left(\sum_{k=1}^{p} a_{3 k} n(s)^{k} W(t)^{k}\right)$

then, we can express the sufficient condition for which the curve $r(s)$ is a line of curvature on the surface $P(s, t)$ as

$$
\begin{aligned}
U\left(t_{0}\right) & =V\left(t_{0}\right)=W\left(t_{0}\right)=0 \text { and } f(0)=g(0)=h(0)=0, \\
\theta(s) & =-\int_{s_{0}}^{s} \tau d s+\theta_{0},
\end{aligned}
$$

$g^{\prime}(0) a_{21} m(s) V^{\prime}\left(t_{0}\right)=-\lambda(s) \sinh \theta, h^{\prime}(0) a_{31} n(s) W^{\prime}\left(t_{0}\right)=-\lambda(s) \cosh \theta$,

$\lambda(s) \neq 0$, where $l(s), m(s), n(s), U(t), V(t)$ and $W(t)$ are $C^{1}$ functions, $a_{i j} \in \mathbb{R}(k=1,2,3 ; j=1,2,3, \ldots, p)$.

Example $7 \operatorname{Let} r(s)=(\cos (s), \sin (s), 0)$ be an arc-length spacelike curve $0<$ $s \leq 2 \pi$.It is easy to show that

$$
\begin{aligned}
& T(s)=(-\sin (s), \cos (s), 0), \\
& N(s)=(-\cos (s),-\sin (s), 0),
\end{aligned}
$$




$$
\begin{aligned}
& B(s)=(0,0,1), \\
& \tau=0 .
\end{aligned}
$$

If we let $\theta(0)=0$, then we have $\theta(s)=0$. By taking $\lambda(s)=s, t_{0}=0$ and the marching-scale functions as

$$
u(s, t)=\sin t, v(s, t)=0, w(s, t)=-\sinh (t s)
$$

then the Eq. (4.6) is satisfied. So, we have the following surface as a member of the surface pencil with common line of curvature $r(s)$ (Fig. 4.1) as

$$
P_{3}(s, t)=(\cos (s)-\sin (t) \sin (s), \sin (s)+\cos (s) \sin (t), \sinh (t s)),
$$

where $0<s \leq 2 \pi,-1 \leq t \leq 1$.

Fig. 4.1. $P_{3}(s, t)$ as a member of the surface pencil and its line of curvature

For the same curve if we choose $t_{0}=0, \lambda(s)=-\cosh (s)$ and the marchingscale functions as

$$
u(s, t)=\sum_{k=1}^{4} \sin ^{k}(s) \sin ^{k}(t), v(s, t)=0, w(s, t)=\sum_{k=1}^{4} \cosh ^{k}(s) \sinh ^{k}(t)
$$

we have the Eqn. (4.5) is satisfied. Thus, the surface

$$
\begin{aligned}
& P_{4}(s, t)=\left(\cos (s)-\sin (s) \sum_{k=1}^{4} \sin ^{k}(s) \sin ^{k}(t), \sin (s)+\cos (s) \sum_{k=1}^{4} \sin ^{k}(s) \sin ^{k}(t),\right. \\
& \left.\sum_{k=1}^{4} \cosh ^{k}(s) \sinh ^{k}(t)\right)
\end{aligned}
$$

is a member of the surface pencil with common line of curvature $r(s)$, where $-1.1 \leq s \leq 1.1, \quad-0.7 \leq t \leq 0.2$ (Fig.4 2). 
Fig. 4.2. $P_{4}(s, t)$ as a member of the surface pencil and its line of curvature

Now let $P=P(s, t)$ be a parametric timelike surface and $r=r(s)$ be a timelike curve. The surface is defined by the given curve as

$$
P(s, t)=r(s)+(u(s, t), v(s, t), w(s, t))\left(\begin{array}{c}
T(s) \\
N(s) \\
B(s)
\end{array}\right)
$$

$L_{1} \leq s \leq L_{2}, T_{1} \leq t \leq T_{2}$.

Let $n_{1} \quad\left(n_{1}=\cos \theta N+\sin \theta B\right)$ be a vector orthogonal to the curve $r(s)$, where $\theta=\theta(s)$ is the Lorentzian spacelike angle between $N$ and $n_{1}$. The curve $r(s)$ is a line of curvature on the surface $P(s, t)$ if and only if $n_{1}$ is parallel to the normal vector $n(s, t)$ of the surface $P(s, t)$ and $S(T)=\omega T, \omega \neq 0$, where $S$ is the shape operator of the surface.

Since the curve $r(s)$ is a parametric curve on the surface $P(s, t)$, there exists 
a parameter $t_{0} \in\left[T_{1}, T_{2}\right]$ such that $P\left(s, t_{0}\right)=r(s) L_{1} \leq s \leq L_{2}$, that is ,

$$
u\left(s, t_{0}\right)=v\left(s, t_{0}\right)=w\left(s, t_{0}\right) \equiv 0, L_{1} \leq s \leq L_{2}, T_{1} \leq t \leq T_{2} .
$$

Fistly, we derive the condition for $n_{1}$ to be parallel to the normal vector $n(s, t)$ of the surface $P(s, t)$ :

The normal vector can be expressed as

$$
\begin{aligned}
& n(s, t)=\frac{\partial P(s, t)}{\partial s} \times \frac{\partial P(s, t)}{\partial t} \\
& =\left(\left(-\tau(s) v(s, t)+\frac{\partial w(s, t)}{\partial s}\right) \frac{\partial v(s, t)}{\partial t}-\right. \\
& \left.\left(\kappa(s) u(s, t)+\tau(s) w(s, t)+\frac{\partial v(s, t)}{\partial s}\right) \frac{\partial w(s, t)}{\partial t}\right) T(s)+ \\
& \left(\left(-\tau(s) v(s, t)+\frac{\partial w(s, t)}{\partial s}\right) \frac{\partial u(s, t)}{\partial t}-\right. \\
& \left.\left(1+\kappa(s) v(s, t)+\frac{\partial u(s, t)}{\partial s}\right) \frac{\partial w(s, t)}{\partial t}\right) N(s)+ \\
& \left(-\left(\kappa(s) u(s, t)+\tau(s) w(s, t)+\frac{\partial v(s, t)}{\partial s}\right) \frac{\partial u(s, t)}{\partial t}+\right. \\
& \left.\left(1+\kappa(s) v(s, t)+\frac{\partial u(s, t)}{\partial s}\right)\left(\frac{\partial v(s, t)}{\partial t}\right)\right) B(s)
\end{aligned}
$$

Thus, we get

$$
n\left(s, t_{0}\right)=\phi_{1}\left(s, t_{0}\right) T(s)+\phi_{2}\left(s, t_{0}\right) N(s)+\phi_{3}\left(s, t_{0}\right) B(s),
$$

where

$$
\begin{aligned}
& \phi_{1}\left(s, t_{0}\right)=\frac{\partial w\left(s, t_{0}\right)}{\partial s} \frac{\partial v\left(s, t_{0}\right)}{\partial t}-\frac{\partial v\left(s, t_{0}\right)}{\partial s} \frac{\partial w\left(s, t_{0}\right)}{\partial t} \\
& \phi_{2}\left(s, t_{0}\right)=\frac{\partial w\left(s, t_{0}\right)}{\partial s} \frac{\partial u\left(s, t_{0}\right)}{\partial t}-\left(1+\frac{\partial u\left(s, t_{0}\right)}{\partial s}\right) \frac{\partial w\left(s, t_{0}\right)}{\partial t}, \\
& \phi_{3}\left(s, t_{0}\right)=\left(1+\frac{\partial u\left(s, t_{0}\right)}{\partial s}\right) \frac{\partial v\left(s, t_{0}\right)}{\partial t}-\frac{\partial v\left(s, t_{0}\right)}{\partial s} \frac{\partial u\left(s, t_{0}\right)}{\partial t} .
\end{aligned}
$$

This follows that $n_{1}(s) / / n\left(s, t_{0}\right), L_{1} \leq s \leq L_{2}$, if and only if there exits a function $\lambda(s) \neq 0$ such that

$$
\phi_{1}\left(s, t_{0}\right)=0, \phi_{2}\left(s, t_{0}\right)=\lambda(s) \cos \theta, \phi_{3}\left(s, t_{0}\right)=\lambda(s) \sin \theta
$$


Secondly, since $S(T)=\omega T, \omega \neq 0$,

$$
\theta(s)=\int_{s_{0}}^{s} \tau d s+\theta_{0}
$$

where $s_{0}$ is the starting value of arc length and $\theta=\theta(s)$. In this paper, we assume that $s_{0}=0$.

Combining (4.8), (4.9) and (4.10), we have the following theorem.

Theorem 8 A timelike curve $r(s)$ is a line of curvature on the surface $P(s, t)$ if and only if the followings are satisfied

$$
\begin{aligned}
& \theta(s)=\int_{s_{0}}^{s} \tau d s+\theta(0), \\
& u\left(s, t_{0}\right)=v\left(s, t_{0}\right)=w\left(s, t_{0}\right) \equiv 0, \\
& \phi_{1}\left(s, t_{0}\right) \equiv 0, \phi_{2}\left(s, t_{0}\right)=\lambda(s) \cos \theta, \phi_{3}\left(s, t_{0}\right)=\lambda(s) \sin \theta .
\end{aligned}
$$

We call the set of surfaces defined by (4.1) - (4.4) or (4-7) - (4-10) timelike surface pencil with a common line of curvature. Any surface $P(s, t)$ satisfying these conditions is a member of this family.

Now, we analyse two different types of the marching-scale functions

$u(s, t), v(s, t)$ and $w(s, t)$ in the Eq. (4.1).

(i) If we choose marching-scale functions as

$$
\begin{aligned}
& u(s, t)=\sum_{k=1}^{p} a_{1 k} l(s)^{k} U(t)^{k}, v(s, t)=\sum_{k=1}^{p} a_{2 k} m(s)^{k} V(t)^{k} \text { and } w(s, t)= \\
& \sum_{k=1}^{p} a_{3 k} n(s)^{k} W(t)^{k}
\end{aligned}
$$

then, we can simply express the sufficient condition for which the curve $r(s)$ is a line of curvature of the surface $P(s, t)$ as 


$$
\begin{aligned}
U\left(t_{0}\right) & =V\left(t_{0}\right)=W\left(t_{0}\right)=0, \\
\theta(s) & =\int_{s_{0}}^{s} \tau d s+\theta, \\
a_{21} m(s) V^{\prime}\left(t_{0}\right) & =\lambda(s) \sin \theta, a_{31} n(s) W^{\prime}\left(t_{0}\right)=-\lambda(s) \cos \theta,
\end{aligned}
$$

$\lambda(s) \neq 0$, where $l(s), m(s), n(s), U(t), V(t)$ and $W(t)$ are $C^{1}$ functions, $a_{i j} \in \mathbb{R}(k=1,2,3 ; j=1,2,3, \ldots, p)$.

(ii) If we choose marching-scale functions as

$$
\begin{aligned}
& u(s, t)=f\left(\sum_{k=1}^{p} a_{1 k} l(s)^{k} U(t)^{k}\right), v(s, t)=g\left(\sum_{k=1}^{p} a_{2 k} m(s)^{k} V(t)^{k}\right) \text { and } \\
& w(s, t)=h\left(\sum_{k=1}^{p} a_{3 k} n(s)^{k} W(t)^{k}\right)
\end{aligned}
$$

then, we can express the sufficient condition for which the curve $r(s)$ is a line of curvature on the surface $P(s, t)$ as

$$
\begin{aligned}
& U\left(t_{0}\right)=V\left(t_{0}\right)=W\left(t_{0}\right)=0 \text { and } f(0)=g(0)=h(0)=0 \\
& \theta(s)=\int_{s_{0}}^{s} \tau d s+\theta_{0}, \\
& g^{\prime}(0) a_{21} m(s) V^{\prime}\left(t_{0}\right)=\lambda(s) \sin \theta, h^{\prime}(0) a_{31} n(s) W^{\prime}\left(t_{0}\right)=-\lambda(s) \cos \theta \\
& \lambda(s) \neq 0, \text { where } l(s), m(s), n(s), U(t), V(t) \text { and } W(t) \text { are } C^{1} \text { functions, } \\
& a_{i j} \in \mathbb{R}(k=1,2,3 ; j=1,2,3, \ldots, p) .
\end{aligned}
$$

Example 9 Let $r(s)=(\cosh (s), 0, \sinh (s))$ be an arc-length timelike curve, 0 $\leq s \leq 2 \pi$.It is easy to show that

$$
\begin{aligned}
& T(s)=(\sinh (s), 0, \cosh (s)), \\
& N(s)=(\cosh (s), 0, \sinh (s)),
\end{aligned}
$$


$B(s)=(0,-1,0)$,

$\tau=0$.

By taking $\lambda(s)=-s, t_{0}=0$ and the marching-scale functions as

$u(s, t)=\sinh (t), v(s, t)=0, w(s, t)=\cosh (s t)$

we have the Eq. (4.12) is satisfied. So, we obtain the following surface as a

member of the surface pencil with common line of curvature $r(s)$ (Fig. 4.3) as

$P_{5}(s, t)=(\cosh (s)+\sinh (t) \sinh (s),-\cosh (s * t), \sinh (s)+\cosh (s) \sinh (t))$,

where $0 \leq s \leq 2 \pi,-1 \leq t \leq 1$.

Fig. 4.3. $P_{5}(s, t)$ as a member of the surface pencil and its line of curvature

For the same curve let $\lambda(s)=-\sinh (s), t_{0}=0$ and the marching-scale functions

$u(s, t)=\sum_{k=1}^{4} \sinh ^{k}(t), v(s, t) \equiv 0, w(s, t)=\sum_{k=1}^{4} \sinh ^{k}(s) \sinh ^{k}(t)$.

Now we have the Eq. (4.11) is satisfied. Thus, the surface 


$$
\begin{aligned}
& \quad P_{6}(s, t)=\left(\cosh (s)+\sinh (s) \sum_{k=1}^{4} \sinh ^{k}(t),-\sum_{k=1}^{4} \sinh ^{k}(s) \sinh ^{k}(t), \sinh (s)+\right. \\
& \left.\cosh (s) \sum_{k=1}^{4} \sinh ^{k}(t)\right) \\
& \quad \text { is a member of the surface pencil with common line of curvature } r(s), \text { where } \\
& -1 \leq s \leq 1,-0.4 \leq t \leq 0.4 .
\end{aligned}
$$

Fig. 4.4. $P_{6}(s, t)$ as a member of the surface pencil and its line of curvature

Acknowledgement 10 The authors appreciate the comments and valuable suggestions of the editor and the reviewer. Their advice helped to improve the clarity and presentation of this paper. The second author would like to thank TUBITAK (The Scientific and Technological Research Council of Turkey) for their financial supports during his doctorate studies.

\section{References}

[1] Wang, G.J., Tang, K., Tai, C.L., Parametric representantion of a surface pencil with a common spatial geodesic, Computer Aided Design, 2004, 36: 447- 
459.

[2] Kasap, E., Akyıldız, F.T., Surfaces with common geodesic in Minkowski 3-space, Applied Mathematics and Computation, 2008, 177: 260-270.

[3] Li, C.Y., Wang, R. H., Zhu, C.G., Parametric representation of a surface pencil with a common line of curvature, Computer Aided Design, 2011, 43: 1110-1117.

[4] Şaffak, G., Kasap, E., Family of surface with a common null geodesic, International Journal of Physical Sciences, 2009, Vol.4 (8): 428-433.

[5] O’Neill, B., Semi-Riemannian Geometry with applications to relativity, New York: Academic Press, 1983.

[6] Akutagawa, K. Nishikawa, S., The Gauss map and spacelike surface with prescribed mean curvature in Minkowski 3-space, Tohoku Math. J., 1990, 42: $67-82$.

[7] Woestijne, V.D.I, Minimal Surface of the 3-Dimensional Minkowski Space, Singapore: World Scientific Publishing, 1990.

[8] Ratcliffe, J.G., Foundations of Hyperbolic Manifolds, New York: SpringerVerlag, 1994.

[9] Beem, J.K., Ehrlich, P.E., Global Lorentzian Geometry, New York: Marcel Dekker, 1981. 